\title{
sciendo
}

\section{Acute Effects of a Static vs. a Dynamic Stretching Warm-up on Repeated-Sprint Performance in Female Handball Players}

\author{
by \\ Piotr Zmijewski ${ }^{1}$ Patrycja Lipinska², Anna Czajkowska , Anna Mróz', \\ Pawet Kapuścinski ${ }^{1}$, Krzysztof Mazurek ${ }^{1}$
}

\begin{abstract}
This randomized cross-over study examined the effects of typical static and dynamic stretching warm-up protocols on repeated-sprint performance. Thirteen young female handball players performed a 5 min aerobic warm-up followed by one of three stretching protocols for the lower limbs: (1) static stretching, (2) dynamic-ballistic stretching, and (3) no stretching before performing five all-out sprints on a cycle ergometer. Each protocol was performed on a different occasion, separated by 2-3 days. Range of movement (ROM) was also measured before and after the warm-up protocols with a sit-and-reach test. Fixed and random effects of each stretching protocol on repeated sprint performance were estimated with mixed linear modeling and data were evaluated via standardization and magnitude-based inferences. In comparison to no stretching, there were small increases in ROM after dynamic stretching $(12.7 \%$, $\pm 0.7 \%$; mean, $\pm 90 \%$ confidence limits) and static stretching $(19.2 \%, \pm 0.9 \%)$. There were small increases in the average power across all sprints with dynamic stretching relative to static stretching $(3.3 \%, \pm 2.4 \%)$ and no stretching $(3.0 \%$, $\pm 2.4 \%)$ and trivial to small increases in the average power in the 1st and 5th trials with dynamic stretching compared to static stretching $(3.9 \%, \pm 2.6 \% ; 2.6 \%, \pm 2.6 \%$, respectively) and no stretching $(2.0 \%, \pm 2.7 \% ; 4.1 \%, \pm 2.8 \%$, respectively). There were also trivial and small decreases in power across all sprints with static relative to dynamic stretching $(-1.3 \%, \pm 2.8 \%)$ and no stretching $(-3.5 \%, \pm 2.9 \%)$. Dynamic stretching improved repeated-sprint performance to a greater extent than static stretching and no stretching.
\end{abstract}

Key words: performance, team sport athletes, high-intensity, modeling.

\section{Introduction}

Team handball is a physically demanding intermittent team sport, where players are exposed to relatively high workloads with substantial aerobic energy expenditure, interspersed with short periods of dominant anaerobic energy production (Michalsik et al., 2013a). A handball match consists of a large number of repeated sprints, accelerations and decelerations, jumps, rapid changes of running direction and contacts, blocking and pushing (Michalsik et al., 2013b). Repeated sprint ability (RSA) is a term used to describe the repetitive application of high-intensity, short duration efforts with recovery periods of varying duration
(Girard et al., 2011). RSA is an important element of fitness, especially in team sports (Gharbi et al., 2015), and it is believed that an appropriate warm-up prior to sprint or repeated sprint exercise can enhance sprint-type performance.

The warm-up is a routine part of training and match preparation in competitive sport and it is often considered a prerequisite for achieving optimal athletic performance. The warm-up effects on performance are determined by several factors, including the rise of core temperature, intensity, duration and the recovery interval between the warm-up and exercise performance, but also the type of exercise (Bishop, 2003).

1 - Jozef Pilsudski University of Physical Education in Warsaw, Warsaw, Poland.

2 - Institute of Physical Education, Kazimierz Wielki University, Bydgoszcz, Poland. 
Consequently, stretching exercises designed to enhance flexibility are regularly included in the training programs of many athletes, along with the pre-event warm-up and activities to re-warm athletes. Warm-ups are typically composed of a sub-maximal aerobic activity, stretching and a sport-specific activity, including high-intensity exercises. The stretching portion traditionally involves static stretching to decrease the risk of muscle-related injuries (Amako et al., 2003), but could itself impair subsequent athletic performance (Behm and Chaouachi, 2011). Although investigations into the effects of static stretching often show some performance impairment versus a dynamic warm-up (McMillian et al., 2006), others have observed no such effect (Little and Williams, 2006). On the other hand, one study indicated that dynamic stretching was not as effective at increasing flexibility as static stretching, thus it may be necessary to include this form of stretching (in a warm-up) for sport-specific purposes (Mikolalec et al., 2012; Wong et al., 2011). Some have observed that stretch-induced reductions in maximal and explosive efforts also play an essential role in handball and similar sports (Cormie et al., 2011; Markovic and Mikulic, 2010). This highlights the need for further studies to determine the optimal warm-up protocols for handball athletes.

Previous studies on the effectiveness of stretching are limited by several factors. First, there are many different warm-up protocols, but few are representative of a typical warm-up methods used by athletes. In practice, handball players usually perform several minutes of running, repeated stretching exercises and some sport-specific activities. Under match conditions, some specific activities are not always possible to conduct. As an example, Beckett et al. (2009) found that the stretching protocols across studies varied considerably, with stretch duration ranging from $90 \mathrm{~s}$ to $30 \mathrm{~min}$ per muscle group. In addition, some static stretching has been conducted alone, without aerobic exercise to supplement the warm-up procedures (Heisey and Kingsley, 2016).

The precise magnitude of stretch-induced changes in repeated sprint performance is still unknown. It would be beneficial for coaches and practitioners to know which type of warm-up protocols is appropriate for a given athlete population and which is most effective in enhancing sport-specific performance. Hence, the purpose of this study was to examine the effects of typical static versus dynamic stretching warmup protocols on measures of RSA taken during a cycle ergometer test. We hypothesized that the static stretching protocol would impair initial power production (i.e., first sprint trial), but it would attenuate the decline in power across following sprint trials due to fatigue.

\section{Methods}

\section{Experimental Approach to the Problem}

This study was designed to address practical requirements of sport concerning the impact of static versus dynamic stretching on RSA performance in well-trained female handball players. A post-only crossover design was employed to examine the effect of a short twophase warm-up (aerobic + stretching) using three different stretching protocols on RSA performance. Participants attended the laboratory on three occasions, each separated by a period of 2-3 days, to minimize the effects of fatigue on performance. On each occasion, participants were required to perform a standardized warm-up supplemented with one of three stretching protocols followed by RSA testing. Before and after the warm-up (within a $90 \mathrm{~s}$ period) a sit-andreach test was applied to evaluate range of motion $(\mathrm{ROM})$ around the hip area. The study design is illustrated in Figure 1. Each athlete was tested at the same time of the day across all trials to control for circadian variability. Participants were asked to maintain their normal physical activity routines and dietary habits across the study. Athletes were familiarized with the RSA and sit-and-reach tests, as well as the warm-up and stretching protocols, before the experiment commenced.

\section{Participants}

Thirteen healthy female athletes (age $=$ $22.1 \pm 3.2$; body height $=1.71 \pm 0.07 \mathrm{~m}$; body mass $=68.5 \pm 10.4 \mathrm{~kg}$, mean $\pm \mathrm{SD}$, respectively) were recruited as participants. All were handball players, who trained three times per week $(1.5-2 \mathrm{~h}$ per session) and were affiliated with a professional club competing in the second division of the Polish academic league. They were assessed at the start of the competitive season after 8 weeks of conditioning. All participants 
were healthy and injury-free at the time of this study. Institutional ethics approval and individual written informed consent were obtained before testing commenced.

\section{Procedures}

Warm-up. Each trial started with a standardized warm-up consisting of general aerobic (cardiovascular) activity and stretching exercises. The aerobic activity consisted of $5 \mathrm{~min}$ of sub-maximal exercise performed on a cycle ergometer (Ergoselect 200, Ergoline GmbH, Germany) with a constant load of $1.5 \mathrm{~W} \cdot \mathrm{kg}^{-1}$. For stretching exercises, one of the three conditions was applied in random order: (1) control (CON) under which participants were required to sit and rest for $6 \mathrm{~min}$, (2) static stretching (SS) under which participants were required to complete a 6 min static stretching protocol, or (3) 6 min of dynamic stretching (DS) exercises. Each of the three experimental trials lasted for around $11 \mathrm{~min}$ in total.

SS protocol. The SS protocol consisted of a total of 12 static stretches (in 3 successive sets) targeting the prime movers of the lower extremities. For the hip extensors and quadriceps, the individual stood in an upright position with one hand against the wall for balance and grasped the ankle with the hand, attempting to fully extend the hip joint (one static stretch for each leg). The hamstrings (hurdler stretch) were targeted in a seated position. The athlete was to extend a single leg and flex the other leg until the foot was in contact with the thigh. The participant flexed forward from the waist, keeping the back flat (one static stretch for each leg). Prior to each stretch, the researcher demonstrated how to properly perform each exercise. All stretches were performed slowly, smoothly and continuously, so that no ballistic, abrupt movements occurred at any time. Each stretch was held to the point of slight discomfort (not pain) for a period of $20 \mathrm{~s}$ per muscle group followed by a $10 \mathrm{~s}$ passive rest period in a neutral position. Each stretch was repeated three times for each limb in alternating order. According to the recommendation (Bandy et al., 1997), it was decided not to increase the stretching duration beyond a single set of $30 \mathrm{~s}$, as it provides no additional increase in ROM.

$D S$ protocol. The DS protocol consisted of a total of 12 sets of leg swings (in 3 successive sets) targeting the same muscle groups, in the same order as the SS protocol, but using dynamic movements instead. This procedure included a set of $20 \mathrm{~s}$ anterior/posterior leg swings in a standing position with the arms in contact with the wall (for stabilization), followed by a $10 \mathrm{~s}$ passive rest interval in a neutral position. As in the SS, each set of stretches (around 14-18 swings in one set/muscle group) was repeated three times for each limb in alternating order.

Sit-and-reach test. A standard sit-and-reach box was placed on the floor. The athlete sat on the floor with their shoes on and fully extended legs so that the soles of the feet were flat against the end of the box. The participant then extended her arms forward, with palms facing down, and reached forward to push the measuring scale as far as possible without bending the knees. Each measurement was repeated twice and the two results were added for analysis.

RSA test. The RSA test was performed on a cycle ergometer (Monark 824E, Sweden). The test protocols consisted of $5 \times 6 \mathrm{~s}$ all-out sprints with $30 \mathrm{~s}$ rest intervals, with the load fixed at $7.5 \%$ of the athlete's body mass. Athletes were instructed to cycle at maximal effort during each $6 \mathrm{~s}$ trial. Strong verbal encouragement was provided during the sprint trials. During the $24 \mathrm{~s}$ recovery period between each trial, participants pedaled slowly without a load. Straps were used to secure the feet to the pedals, and each sprint was performed in a seated position. MCE software (Version 5.1, JBAZb. Staniak, Poland) was used to calculate the following performance variables: average power across all sprints, average power in the $1^{\text {st }}$ and $5^{\text {th }}$ sprints, and power drop-off across all five trials (fatigue after 5 sprints).

\section{Statistical analysis}

Changes and differences in ROM across the three warm-up protocols were examined using a spreadsheet for a post-only crossover trial (Hopkins, 2017). The effects were interpreted using magnitude-based inferences (Hopkins et al., 2009). Mixed linear modeling, performed using Proc Mixed in the Statistical Analysis System (version 9.4, SAS Institute, Cary NC, USA), was used to evaluate the RSA components. The fixed effect was the type of stretching (to estimate the means for each protocol and the mean difference between them) analyzed for average power across all sprints, average power in the $1^{\text {st }}$ and $5^{\text {th }}$ sprints, 
and declining power across all sprints. The random effects were: the identity of each athlete (real between-athlete difference in overall variability, representing how much faster or slower they were on average); the interaction of repetition with identity of the athlete (withinsubject variability) representing individual differences of each athlete of the mean slopes in the five sprints; the interaction of the type of stretching with athlete identity (a response differentiation for different types of stretching), representing the random error arising from the athlete in each type of stretching; the interaction of the last sprint with identity of the athlete, representing the athlete's way of dealing with the last sprint - either faster or slower than the mean reduction; and the residual random (typical) error that accompanied every sprint. The effects were interpreted using magnitude-based inferences (Hopkins et al., 2009).

All data were log-transformed to reduce the bias arising from non-uniformity error. Means of the score changes, SDs of the score changes, and effects (differences in the changes of the means and their certainty limits) were backtransformed to percentage units. The smallest important difference, to assess all effects, was 0.2 of the observed between-athlete SD. That SD was derived by adding up all the variances that contributed to a single sprint in any given testing session - here the real between-athlete differences in overall variability plus the variance an athlete showed in any given stretching protocol (set-toset within-subject variability), plus the sprint-tosprint random typical error. Threshold values for assessing magnitudes of standardized effects were $0.20,0.60,1.2,2.0$ and 4.0 for small, moderate, large, very large and extremely large effects, respectively. To interpret SDs of two random effects, set-to-set within-subject variability and sprint-to-sprint within-subject variability, thresholds were halved. Uncertainty in each effect was expressed as a $90 \%$ confidence limit, as well as a probability of the true effect being substantially positive (an increase) or negative (a decrease). These probabilities were used to make a qualitative, probabilistic, nonclinical inference about the true effect: if the probability of the effect being a substantial increase or decrease was $>5 \%$ in both cases (equivalent to the $90 \%$ confidence interval overlapping thresholds for a substantial increase and decrease), the effect was reported as unclear; otherwise, it was considered clear and assigned to the relevant magnitude value, with the qualitative probability of the true effect being a substantial increase, substantial decrease, or a trivial difference (whichever outcome had the largest probability). The following scale for interpreting the probabilities was used: $25-75 \%$, possible; 75-95\%, likely; 95-99.5\%, very likely; $>99.5 \%$, most likely.

\section{Results}

Baseline values for the sit-and-reach tests across the CON, SS and DS conditions were $12.2 \pm$ $0.6 \mathrm{~cm}, 11.9 \pm 0.5 \mathrm{~cm}$ and $12.4 \pm 0.6 \mathrm{~cm}$, respectively (mean $\pm \mathrm{SD}$ ). Athletes demonstrated an increase in ROM for the DS and SS protocols by 12.7 and $19.2 \%$ (90\% confidence limits \pm 0.7 and $\pm 0.9 \%$, both small differences, possible and very likely), while the corresponding change for CON was trivial. Simple statistics of fixed effects for different stretching protocols, analyzed for average power across all sprints, average power in the $1^{\text {st }}$ sprint, average power in the $5^{\text {th }}$ sprint, and drop in power across all sprints (fatigue after 5 sprints) are presented in Table 1 . The highest average power across all sprints was produced after DS in comparison to SS and CON. We also observed higher average power in the $1^{\text {st }}$ and $5^{\text {th }}$ sprints for DS. After SS the average power was smaller than under the CON condition. Only power drop across 5 sprints was slightly smaller after SS. Figure 2 presents lines of power decrement across five sprints for every type of stretching depending on the warm-up protocol.

Table 2 shows all random effects as SDs and their magnitudes in relation to thresholds for moderate and large effects. We observed large individual differences in fatigue over the 5 sprint trials. Individual responses to the different stretching protocols were moderate in magnitude, while the individual difference in the $5^{\text {th }}$ trial was moderate. Random (typical) error which accompanied every sprint was also moderate.

Percent differences in the means of all measured components of RSA between stretching protocols and their magnitudes in relation to thresholds for trivial and small effects are presented in Table 3. Dynamic stretching caused substantial effects in average power across all sprints and in average power in the $1^{\text {st }}$ and $5^{\text {th }}$ 
sprint. Effects were small in magnitude, but clear. Power declined across the 5 sprints with the smallest magnitude after SS, but the difference in comparison to DS was trivial.

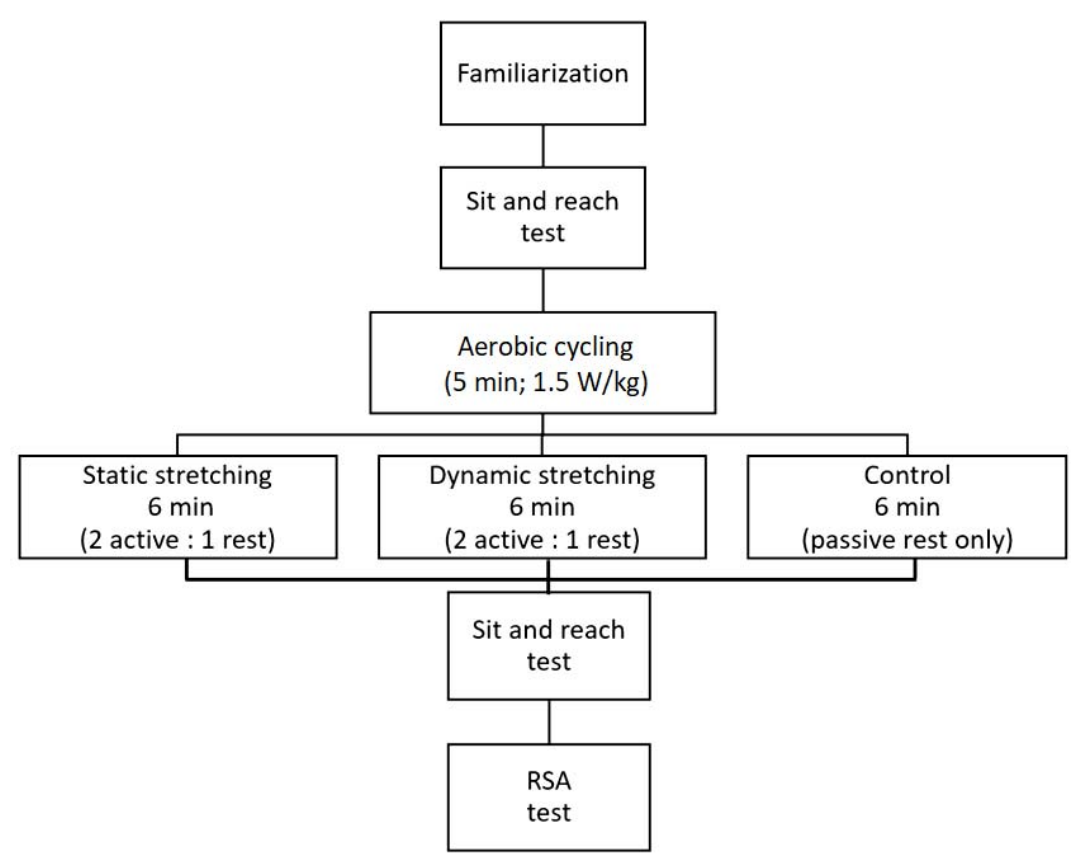

Figure 1

Flow chart illustrating the research design and randomly-ordered conditions.

$R S A$ - repeated sprint ability

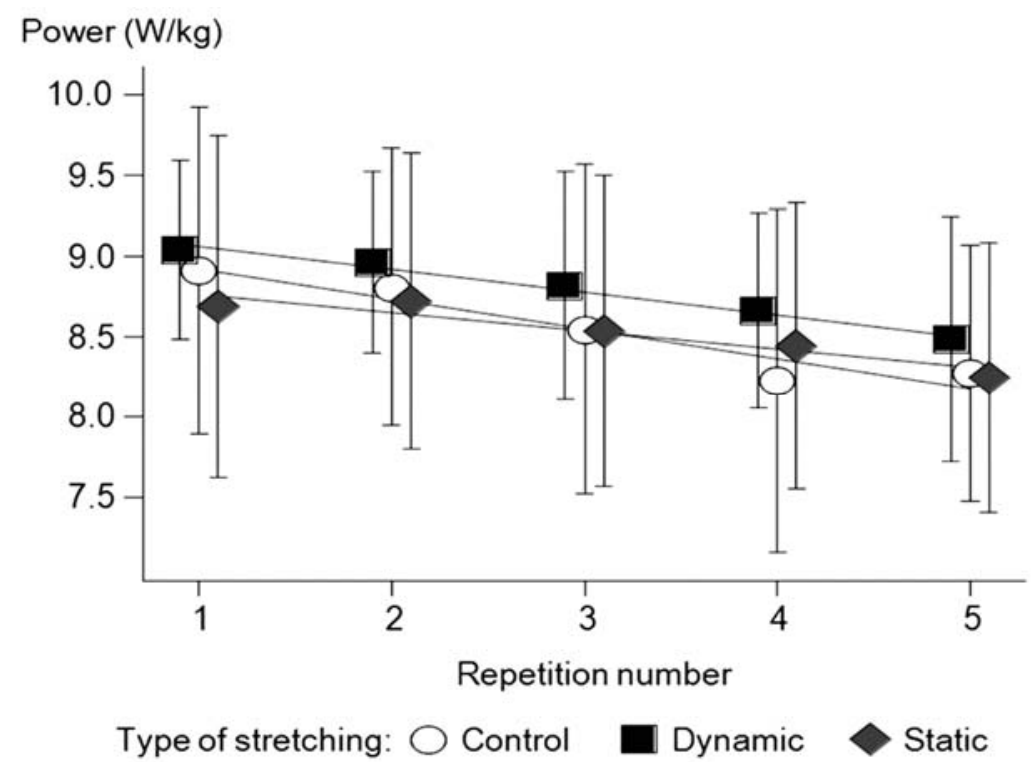

Figure 2

Power drop across five sprints for every type of the stretching protocol 


\section{Table 1}

Basic statistics for average power maintained with three types of stretching.

Data are mean $\pm S D$ in columns $2-4$ and mean, $\pm 90 \% C L(90 \%$ confidence limits in \pm form) in the last column.

\begin{tabular}{|c|c|c|c|c|}
\hline $\begin{array}{l}\text { Type of } \\
\text { stretching }\end{array}$ & $\begin{array}{l}\text { Average power across } \\
\text { all sprints }(\mathrm{W} / \mathrm{kg})\end{array}$ & $\begin{array}{c}\text { Average power in } \\
\text { the } \\
1 \text { st sprint }(\mathrm{W} / \mathrm{kg})\end{array}$ & $\begin{array}{c}\text { Average power in } \\
\text { the } 5^{\text {th }} \text { sprint } \\
(\mathrm{W} / \mathrm{kg})\end{array}$ & $\begin{array}{c}\text { Power drop across } \\
5 \text { sprints (\%) }\end{array}$ \\
\hline Control & $8.55 \pm 0.96$ & $8.91 \pm 1.02$ & $8.27 \pm 0.80$ & $-8.5, \pm 3.5$ \\
\hline Dynamic & $8.79 \pm 0.65$ & $9.04 \pm 0.55$ & $8.49 \pm 0.76$ & $-6.6, \pm 3.5$ \\
\hline Static & $8.53 \pm 0.93$ & $8.69 \pm 1.06$ & $8.25 \pm 0.84$ & $-5.3, \pm 3.6$ \\
\hline
\end{tabular}

Table 2

Standard deviations $(S D)$ representing random effects: real between-athletes differences, individual differences in fatigue over 5 sprints, within-subject random variability, individual differences in extra effort in the $5^{\text {th }}$ sprint and the observed sprintto-sprint random error for average power. All data are percents $\pm 90 \% C L$

\begin{tabular}{|c|c|c|}
\hline & $\mathrm{SD}, \pm 90 \% \mathrm{CL}$ & Inference ${ }^{a}$ \\
\hline Real between-athlete difference & $9.7, \pm 3.8$ & - \\
\hline $\begin{array}{l}\text { Individual difference in fatigue over } 5 \text { sprints (sprint-to } \\
\text { sprint within-subject variability) }\end{array}$ & $6.7, \pm 3.3$ & large \\
\hline $\begin{array}{l}\text { Within-athlete Stretching-protocol-to-Stretching-protocol } \\
\text { variability }\end{array}$ & $3.1, \pm 1,0$ & moderate \\
\hline Individual difference in extra effort in the $5^{\text {th }}$ sprint & $3.0, \pm 3.3$ & moderate \\
\hline Sprint-to-sprint random error & $3.5, \pm 0.4$ & moderate \\
\hline \multicolumn{3}{|c|}{$\begin{array}{l} \pm 90 \% C L, 90 \% \text { confidence limits in } \pm \text { form. } \\
\text { aMagnitude of the sample SD in relation to thresholds for moderate and large of } \\
3.15 \% \text { and } 6.41 \% \text { respectively ( } 0.3 \text { and } 0.6 \text { of the observed between-athlete SD, } \\
\text { see Methods). }\end{array}$} \\
\hline
\end{tabular}


Table 3

Magnitude-based inferences for differences in the means. Data are percents, $\pm 90 \% C L$

\begin{tabular}{lcc} 
Type of stretching & Mean $^{\mathrm{a}}, \pm 90 \% \mathrm{CL}$ & Inference $^{\mathrm{a}}$ \\
\hline \multicolumn{2}{r}{ Average power across all sprints } & \\
Dynamic vs. Control & $3.0, \pm 2.4$ & small $\uparrow^{*}$ \\
Dynamic vs. Static & $3.3, \pm 2.4$ & small $\downarrow^{* *}$ \\
Static vs. Control & $-0.3, \pm 2.4$ & trivial $^{* *}$
\end{tabular}

Average powerin the $1^{\text {st }}$ sprint

$\begin{array}{ccc}\text { Dynamic vs. Control } & 2.0, \pm 2.7 & \text { trivial to small } \uparrow^{*} \\ \text { Dynamic vs. Static } & 3.9, \pm 2.6 & \text { small } \downarrow^{* *} \\ \text { Static vs. Control } & -2.0, \pm 2.6 & \text { trivial to small } \downarrow^{*}\end{array}$

Average power in the $5^{\text {th }}$ sprint

$\begin{array}{ccc}\text { Dynamic vs. Control } & 4.1, \pm 2.8 & \text { small } \uparrow * * \\ \text { Dynamic vs. Static } & 2.6, \pm 2.6 & \text { small } \downarrow * \\ \text { Static vs. Control } & 1.4, \pm 2.7 & \text { trivial } \uparrow *\end{array}$

Power drop across 5 sprints (fatigue after 5 sprints)

Dynamic vs. Control

$2.1, \pm 2.9$

small $\uparrow *$

Dynamic vs. Static

$-1.3, \pm 2.8$

trivial $\uparrow^{*}$

Static vs. Control

$3.5, \pm 2.9$

small $\uparrow^{* *}$

$\pm 90 \% \mathrm{CL}, 90 \%$ confidence limits in \pm form.

aMagnitude thresholds (for percent difference in means): <2.09, trivial; 2.10-6.40,

small (of 0.2 of the observed between-athlete SD as the smallest important

difference). Asterisks indicate effects clear at the 5\% level and likelihood that the true effect is substantial, as follows: *possible, **ikely.

\section{Discussion}

The most important finding of the present study was that DS during the warm-up can enhance repeated sprint performance among female handball players.

This was achieved with a warm-up routine comprising $5 \mathrm{~min}$ of aerobic exercise with 6 min of DS, which also presented a more beneficial stimulus for repeated sprint performance than either $5 \mathrm{~min}$ of aerobic exercise supplemented with $6 \mathrm{~min}$ of SS or a $6 \mathrm{~min}$ passive rest protocol. The observed effects were small yet substantial $(3.3 \%$ and $3.0 \%$, respectively). Other authors demonstrated that an $0.8 \%$ improvement in sprinting, in team sports is the smallest significant effect (Paton et., 2001). We found that effects of DS on repeated sprint performance, in comparison to SS, ranged from 2.6 to $3.9 \%$, thus the improvements were much higher than the smallest worthwhile change.

It was anticipated that the SS protocol would impair average power in the first sprint, but maintain power (i.e. attenuate the decline in power) across subsequent sprint trials. We noted higher average power in the $1^{\text {st }}$ and the $5^{\text {th }}$ sprint after DS than after CON and SS. The SS protocol 
had a substantial small negative effect on average power in the first sprint when compared to the DS protocol $(-3.9 \%)$ and a trivial to small effect versus CON $(-2.0 \%)$. Also, we did not find a positive effect of SS on power in the $5^{\text {th }}$ sprint from the DS condition. Notably, the DS protocol also increased power in the $5^{\text {th }}$ sprint, when compared to CON and SS. A greater positive effect on average power in the $5^{\text {th }}$ sprint was observed after DS vs. CON $(4.1 \%$, small substantial effect) than DS vs. SS (2.6\%, small substantial effect). In addition, when power was evaluated across all sprints (fatigue after 5 sprints), the SS protocol caused smaller decreases compared to DS (-1.3\%, trivial effect) and to $\mathrm{CON}$ ( $3.5 \%$, small effect).

To evaluate the means for each type of stretching and the mean difference between them a novel approach in this study was used, i.e., mixed linear modeling, which, apart from calculating fixed effects described above, estimated random effects. It allowed us to determine between-athlete SD and then the smallest significant difference in power measurements between different types of stretching protocols. The real between-athlete difference representing how much faster or slower they were on average was $9.7 \%( \pm 3.8 \%$, $90 \%$ CL), with different training experience and positions among the players. Individual differences in fatigue over 5 sprints representing individual differences of each athlete of the mean slope in the five sprints were large $(6.7 \%, \pm 3.3 \%)$, which showed that the tested group was diverse in terms of overall physical ability resulting presumably from training, positional and genetic factors (e.g. muscle fiber variability). The withinathlete difference representing a response for different types of stretching was moderate in magnitude $(3.1 \%, \pm 1.0 \%)$, presumably resulting from recent exercise/training performed by the athlete. Menstrual cycle fluctuations could conceivably explain some of this variability, but we were unable to control for this variability during the competitive season. Individual difference in the $5^{\text {th }}$ sprint, representing the athlete's way of dealing with the last sprint, was moderate $(3.0 \%, \pm 3.4 \%)$ and can be explained by the individual approach of the athletes to the last sprint: some applied maximal effort and others held back. To our knowledge, this is one of the first studies to specifically compare effects of SS versus DS (as part of a warm-up) on repeated sprint cycling performance in competitive female athletes. Previous researchers examining SS have focused on a single sprint, jumping ability or isolated movements. A similar study by Taylor et al. (2013) was conducted on sub-elite male soccer players. This cohort completed a warm-up protocol that started with $5 \mathrm{~min}$ of jogging, followed by no stretching, SS or DS, and finished with a task-specific high-intensity activity. They reported the fastest sprint times and higher posttest blood lactate after the 2-phase warm-up, compared with a 3-phase warm-up that included SS. In another study, $20 \mathrm{~m}$ RSA was compromised when SS was conducted after dynamic activities (Sim et al., 2009). These findings are supported by other studies, where the counterbalancing of static and dynamic-type stretches on RSA was reported (Wong et al., 2011). Haddad et al. (2014) analyzed the effects of SS on RSA, but using a $15 \mathrm{~min}$ protocol and $24 \mathrm{~h}$ rest period. They found that SS of the lower limbs and hip muscles had a negative effect on explosive performance for up to 24 hours, but with no major effects on RSA. We designed our study to address the practical necessities of sport, thus it is not possible to generalize the above results due to major differences in the study design.

We also analyzed the effects of SS versus DS on single $\left(1^{\text {st }}\right)$ sprint performance. Our results support previous investigations, whereby SS impaired maximal power during a $6 \mathrm{~s}$ cycling sprint compared to DS and CON. This finding corroborates a study on male rugby union players, where passive stretching caused significantly slower $20 \mathrm{~m}$ sprint times (Fletcher and Jones, 2004). Similarly, in elite female soccer players, decreases in acceleration, maximalvelocity sprint time and overall sprint time were observed after the SS routine versus a nostretching warm-up (Sayers et al., 2008). Others reported significantly slower $20 \mathrm{~m}$ sprint times after four sets of three passive stretches each lasting $30 \mathrm{~s}$, as compared with a no-stretching condition (Nelson et al., 2005). Some studies have reported improvements in performance. Gonzalez-Rave et al. (2009) examined the acute effects of heavy-load resistance exercises, SS exercises, and heavy-load plus SS exercises in untrained subjects (González-Ravé et al., 2009). Only the stretching exercises produced an 
increase in squat jump and counter-movement jump performance. Elsewhere, it was reported that a warm-up including SS can benefit anaerobic power in comparison to sub-maximal cycling alone ( $\mathrm{O}^{\prime}$ Connor et al., 2006). Haag et al. (2010) also investigated the effects of acute SS on pitching velocity and accuracy of baseball players, reporting no negative effect on pitching performance. Others have reported no reduction in strength, power or explosive muscular performance following SS (Simic et al., 2013). Some authors suggest that highly trained individuals are more resistant to these stretchinduced deficits (Chaouachi et al., 2010; Little and Williams, 2006). In a literature review, it was shown that an acute bout of SS generally decreases maximal muscle strength, muscle power, and explosive muscular performance by $5.4 \%$ (95\% CI: -6.6 to $-4.2 \%$ ), $-1.9 \%$ (95\% CI: -4.0 to $0.2 \%)$, and $-2.0 \% \quad(95 \%$ CI: -2.8 to $-1.3 \%)$, respectively (Simic et al., 2013).

In contrast to the performance impairment resulting from SS, a number of DS protocols have been found effective in facilitating subsequent exercise performance (Wong et al., 2011). Dynamic stretching was the most favorable approach in this study. Specifically, we found small, but significant improvements in single and repeated sprint performance after the DS protocol, when compared to CON and SS. This is in agreement with the majority of studies in this area (Behm et al., 2016; Behm and Chaouachi, 2011; Peck et al., 2014). Some research has reported no effect from DS on indices of performance (Chaouachi et al., 2010; Samuel et al., 2008). Following reviews of the literature, it was concluded that DS generally leads to facilitation of power, sprint and jump performance, or at least no adverse effect (Behm and Chaouachi, 2011; Peck et al., 2014). In the present study, the DS protocol was the most beneficial for peak power in the $1^{\text {st }}$ sprint, $5^{\text {th }}$ sprint and total repeated sprint performance (vs. SS). These results suggest that it may be preferable to avoid SS before repeated sprint performance. Turki et al. (2011) stated that the positive effects of DS prior to power performance could be explained by several factors: an elevated heart rate and muscle and body temperature, enhanced motor unit excitability and better kinesthetic sense.

Some hypotheses have also been discussed to explain the negative effects of stretching on the neuromuscular system (Behm et al., 2011). Trajano et al. (2017) proposed that inhibition occurring at the motoneuronal level after passive muscle stretch is a major factor affecting the neural efferent drive to the muscle and, subsequently, its ability to produce maximal force. It was also proposed that muscle stretch could influence cortical activity, as the muscle spindle (i.e. stretch-activated) type I and II afferent fibres project to cortical areas 3a (somatosensory cortex) and 4 (motor cortex), which provides evidence for the possibility that muscle stretch could influence cortical activity. Neurons in the cortical region can affect spinal motor neurons of the stretched muscles via interneurons (Trajano et al., 2017). The reduced spinal excitability and impaired motor neuron facilitation processes are currently perceived as significant candidates to explain force loss after static stretching exercise (Trajano et al., 2017). The negative effect of SS occurs regardless of the subject's age, gender or training status, while the corresponding acute effects of SS on muscle power are still unclear (Simic et al., 2013). However, the acute effects of SS on maximal muscular performance are task-specific, with the type of muscle contraction being an important factor (Simic et al., 2013).

Another important factor is the intensity and duration of SS. It was shown that a warm-up supplemented with short-duration (i.e., 20 s) SS of quadriceps and hamstring muscles can increase ROM and did not negatively influence sprint performance compared with a sprint-only training program (Chaouachi et al., 2008). In our study, we also used $20 \mathrm{~s}$ duration of SS, but the detrimental effect of SS versus DS on sprint performance was clear, probably owing to the stretching duration being more than double in total. Given the existing methodological differences, accurate comparisons between this and other study results are somewhat limited. We also analyzed females only, but some studies have shown that men tend to exhibit greater leg stiffness (Blackburn et al., 2009).

Though some authors conclude that SS prior to training or competition may not be detrimental to athletic performance (de Oliveira and Rama, 2016), this form of stretching is generally avoided prior to high-power and 
explosive-strength performance (Sim et al., 2009). On the other hand, the need to enhance performance should also be balanced against injury prevention (Young, 2007), as SS is recognized as more effective in increasing ROM than DS, thereby it reduces the risk of muscle injury. Thus, combining both SS and DS in a single warm-up protocol could justify and address these needs. Appropriate DS can provide similar increases in static flexibility as SS (Beedle and Mann, 2007), although performing dynamic activities after stretching may attenuate any potential impairment in sprint ability resulting from SS. One recommendation is to avoid an SS protocol alone, acutely prior to high-intensity or explosive exercise performance. A warm-up should contain dynamic activities alone (no SS) or SS should be followed by dynamic activities of moderate duration. This study was designed to address practical necessities of sport concerning the impact of SS versus DS on RSA performance in well-trained female handball players. A warmup routine containing dynamic activities alone (no SS) or SS followed by dynamic activities of moderate duration (5-10 $\mathrm{min})$ may be the preferred warm-up for team sport athletes, as compared to a warm-up during which dynamic activities are followed by SS (Behm and Chaouachi, 2011; Sim et al., 2009).

One limitation of our study includes the inability to control for pre-season conditioning in the tested athletes. Furthermore, this was a nonblinded study, as the coaches and student-athletes were aware of the stretching protocol they were randomized to. the athletes were accustomed to physical testing, differences in their experience and playing position could introduce some uncontrolled learning effect or other unforeseen effects. Another limitation is the lack of control regarding menstrual cycle fluctuations. To control for the most important conditions (execution of the RSA test, ground type, precision of measurement), we also conducted the study in the laboratory (non-specific task for athletes) and not under natural field conditions. Finally, the results obtained under laboratory conditions do not always transfer well to actual sporting performance, and thus our findings should be interpreted and applied with caution. In this study, a limitation is the non-specific form of evaluating power (or speed) for team sport players, as cycling eliminates to a large extent the SSC and thus, it is difficult to induce mechanical changes in muscle due to stretching or resistance exercise. Thus, usually multiple sprints with a change of direction are the more recommended form of testing exercise for team sport athletes.

The results of this study support the use of DS in a warm-up as a strategy to avoid a decrease in power production during repeated cycling sprints, while the same portion of SS provided no such benefits. This study provides evidence that a short, well-designed warm-up routine can optimize RSA in well-trained female athletes.

\section{Practical Implications}

Practitioners involved in female team sports or individual sports where power is of paramount importance, should consider employing DS protocols prior to selected training sessions and competition to improve repeated sprint performance. Within a warm-up routine, it would be useful to include several minutes of DS of the lower limbs (ideally based on sport-specific requirements) after a short period of aerobic exercise which increases body temperature and circulation.

\section{References}

Amako M, Oda T, Masuoka K, Yokoi H, Campisi P. Effect of static stretching on prevention of injuries for military recruits. Mil Med, 2003; 168(6): 442-6

Bandy WD, Irion JM, Briggler M. The effect of time and frequency of static stretching on flexibility of the hamstring muscles. PhysTher, 1997; 77(10): 1090-6

Beckett JRJ, Schneiker KT, Wallman KE, Dawson BT, Guelfi KJ. Effects of Static Stretching on Repeated Sprint and Change of Direction Performance. Med Sci Sport Exerc, 2009; 41(2): 444-5

Beedle BB, Mann CL. A comparison of two warm-ups on joint range of motion. J Strength Cond Res, 2007; 21(3): 776 
Behm DG, Blazevich AJ, Kay AD, McHugh M. Acute effects of muscle stretching on physical performance, range of motion, and injury incidence in healthy active individuals: a systematic review. Appl Physiol Nutr Metab. 2016; 41(1): 1-11

Behm DG, Chaouachi A. A review of the acute effects of static and dynamic stretching on performance. Eur J Appl Physiol, 2011; 111(11): 2633-51

Behm DG, Plewe S, Grage P, Rabbani A, BeigiHT, Byrne JM, Button DC. Relative static stretch-induced impairments and dynamic stretch-induced enhancements are similar in young and middle-aged men. Appl Physiol Nutr Metab, 2011; 36(6): 790-7

Bishop D. Warm up II: performance changes following active warm up and how to structure the warm up. Sports Med, 2003; 33(7): 483-98

Blackburn J, Bell DR, Norcross MF, Hudson JD, Engstrom LA. Comparison of hamstring neuromechanical properties between healthy males and females and the influence of musculotendinous stiffness. $J$ Electromyogr Kinesiol, 2009; 19(5): e362-9

Chaouachi A, Castagna C, Chtara M, Brughelli M, Turki O, Galy O, Chamari K, Behm DG. Effect of WarmUps Involving Static or Dynamic Stretching on Agility, Sprinting, and Jumping Performance in Trained Individuals. J Strength Cond Res, 2010; 24(8): 2001-11

Chaouachi A, Chamari K, Wong P, Castagna C, Chaouachi M, Moussa-Chamari I, Behm DG. Stretch and sprint training reduces stretch-induced sprint performance deficits in 13- to 15-year-old youth. Eur J Appl Physiol, 2008; 104(3): 515-22

Cormie P, McGuiganMR, Newton RU. Developing Maximal Neuromuscular Power. Sport Med, 2011; 41(1): 17-38

de Oliveira FCL, Rama LMPL. Static stretching does not reduce variability, jump and speed performance. Int J Sports Phys Ther, 2016; 11(2): 237-46

Fletcher IM, Jones B. The effect of different warm-up stretch protocols on 20 meter sprint performance in trained rugby union players. J Strength Cond Res, 2004; 18(4): 885-8

Gharbi Z, Dardouri W, Haj-Sassi R, Chamari K, Souissi N. Aerobic and anaerobic determinants of repeated sprint ability in team sports athletes. Biol Sport, 2015; 32(3): 207-12

Girard O, Mendez-Villanueva A, Bishop D. Repeated-Sprint Ability - Part I. Sport Med, 2011; 41(8): 673-94

González-Ravé JM, Machado L, Navarro-Valdivielso F, Vilas-Boas JP. Acute effects of heavy-load exercises, stretching exercises, and heavy-load plus stretching exercises on squat jump and countermovement jump performance. J Strength Cond Res, 2009; 23(2): 472-9

Haag SJ, Wright GA, Gillette CM, Greany JF. Effects of acute static stretching of the throwing shoulder on pitching performance of national collegiate athletic association division III baseball players. J strength Cond Res, 2010; 24(2): 452-7

Haddad M, Dridi A, Chtara M, Chaouachi A, Wong DP, Behm D, Chamari K. Static Stretching Can Impair Explosive Performance for At Least 24 Hours. J Strength Cond Res, 2014; 28(1): 140-6

Heisey CF, Kingsley JD. Effects of Static Stretching on Squat Performance in Division I Female Athletes. Int J Exerc Sci, 2016; 9(3): 359-67

Hopkins WG, Marshall SW, Batterham AM, Hanin J. Progressive Statistics for Studies in Sports Medicine and Exercise Science. Med Sci Sport Exerc, 2009; 41(1): 3-13

Hopkins WG. Spreadsheets for analysis of controlled trials, crossovers and time series. Sportscience, 2017; 21: $1-5$

Little T, Williams AG. Effects of differential stretching protocols during warm-ups on high-speed motor capacities in professional soccer players. J Strength Cond Res, 2006; 20(1): 203-7

Markovic G, Mikulic P. Neuro-Musculoskeletal and Performance Adaptations to Lower-Extremity Plyometric Training. Sport Med, 2010; 40(10): 859-95

McMillian DJ, Moore JH, Hatler BS, Taylor DC. Dynamic versus static - stretching warm-up: the effect on power and agility performance. J Strength Cond Res, 2006; 20(3): 492-9

Michalsik L, Madsen K, Aagaard P. Match performance and physiological capacity of female elite team handball players. Int J Sports Med, 2013; 35(07): 595-607 
Michalsik LB, Aagaard P, Madsen K. Locomotion characteristics and match-induced impairments in physical performance in male elite team handball players. Int J Sports Med, 2013; 34(7): 590-9

Mikolajec K, Waskiewicza Z, Maszczyk A, Bacik B, Kurek P, Zajac A. Effects of stretching and strength exercises on speed and power abilities in male basketball players. Isokinet Exerc Sci, 2012; 20: 61-69

Nelson AG, Driscoll NM, Landin DK, Young MA, Schexnayder IC. Acute effects of passive muscle stretching on sprint performance. J Sports Sci, 2005; 23(5): 449-54

O'Connor DM, Crowe MJ, Spinks WL. Effects of static stretching on leg power during cycling. J Sports Med Phys Fitness, 2006; 46(1): 52-6

Paton CD, Hopkins WG, Vollebregt L. Little effect of caffeine ingestion on repeated sprints in team-sport athletes. Med Sci Sports Exerc, 2001; 33(5): 822-5

Peck E, Chomko G, Gaz DV, Farrell AM. The effects of stretching on performance. Curr Sports Med Rep, 2014; 13(3)

Samuel MN, Holcomb WR, Guadagnoli MA, Rubley MD, Wallmann H. Acute Effects of Static and Ballistic Stretching on Measures of Strength and Power. J Strength Cond Res, 2008; 22(5): 1422-8

Sayers AL, Farley RS, Fuller DK, Jubenville CB, Caputo JL. The Effect of Static Stretching on Phases of Sprint Performance in Elite Soccer Players. J Strength Cond Res, 2008; 22(5): 1416-21

Sim AY, Dawson BT, Guelfi KJ, WallmanKE, Young WB. Effects of Static Stretching in Warm-Up on Repeated Sprint Performance. J Strength Cond Res, 2009; 23(7): 2155-62

Simic L, Sarabon N, Markovic G. Does pre-exercise static stretching inhibit maximal muscular performance? A meta-analytical review. Scand J Med Sci Sport, 2013; 23(2): 131-48

Taylor JM, Weston M, Portas MD. The effect of a short practical warm-up protocol on repeated sprint performance. J Strength Cond Res, 2013; 27(7): 2034-8

Trajano GS, Nosaka K, Blazevich AJ. Neurophysiological Mechanisms Underpinning Stretch-Induced Force Loss. Sports Med, 2017; 47: 1531-1541

Turki O, Chaouachi A, Drinkwater EJ, Chtara M, Chamari K, Amri M, Behm DG. Ten Minutes of Dynamic Stretching Is Sufficient to Potentiate Vertical Jump Performance Characteristics. J Strength Cond Res, 2011; 25(9): 2453-63

Wong DP, Chaouachi A, Lau PWC, Behm DG. Short Durations of Static Stretching when Combined with Dynamic Stretching do not Impair Repeated Sprints and Agility. J Sports Sci Med, 2011; 10(2): 408-16

Young WB. The use of static stretching in warm-up for training and competition. Int J Sports Physiol Perform, 2007; 2(2): 212-6

\section{Corresponding author:}

\section{Piotr Żmijewski}

Faculty of Physical Education

Jozef Pilsudski Academy of Physical Education in Warsaw, Warsaw, Poland, ul. Marymoncka 34,

00-968 Warszawa

E-mail: zmijewski@op.pl 